Supporting information for

\title{
Concentration effects on the first reduction process of methyl viologens and diquat redox flow battery electrolytes
}

\author{
Authors \\ Eduardo Martínez-González ${ }^{\dagger}$, Martha M. Flores-Leonar`, Carlos Amador-Bedolla ${ }^{\dagger}$, \\ Víctor M. Ugalde-Saldívar ${ }^{*} \dagger$ \\ †Facultad de Química, Universidad Nacional Autónoma de México, Ciudad de México, 04510, \\ México. \\ tDepartment of Chemistry, University of Toronto, Toronto, ON, M5S 3H6, Canada. \\ E-mail: vmus@unam.mx
}

This article is dedicated to the memory of Dr. José de Jesús García Valdés, researcher at Facultad de Quimica, UNAM. An excellent colleague affected by this pandemic.

\section{Table of contents}

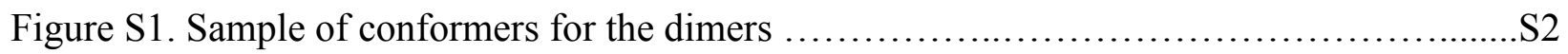

Table $\mathrm{S} 1$. Interaction energies in $\mathrm{kcal} \mathrm{mol}^{-1}$ calculated from single-point energies $\left(\Delta \mathrm{E}_{\mathrm{SP}}\right) \ldots \ldots \ldots \ldots . \mathrm{S} 3$ Table S2. Interaction energies optimized $\left(\Delta \mathrm{E}_{\mathrm{OPT}}\right)$, and calibrated using the $\mathbf{D Q}^{+\bullet}$ pimer $\left(\Delta \mathrm{E}_{\mathrm{CAL}}\right)$ 
a) $\left[\mathrm{MV}^{\cdot+}\right]_{2}$
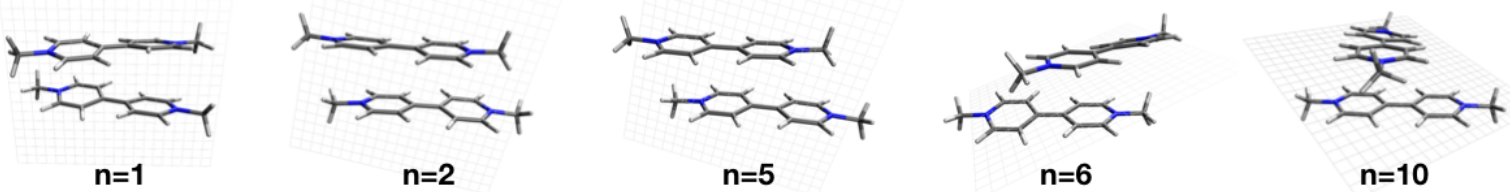

b) $\left[(\mathrm{NPr})_{2}^{2+}-\mathrm{Vi}^{-+}\right]_{2}$
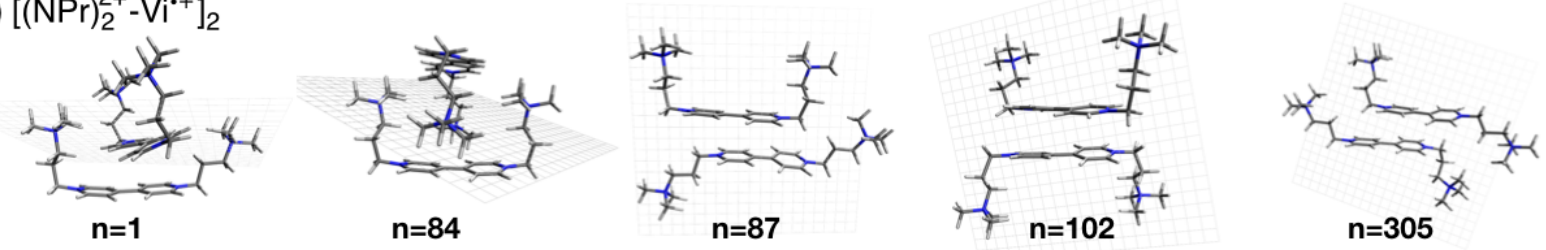

c) $\left[(\mathrm{SPr})_{2}^{2-}-\mathrm{Vi}^{\cdot+}\right]_{2}$
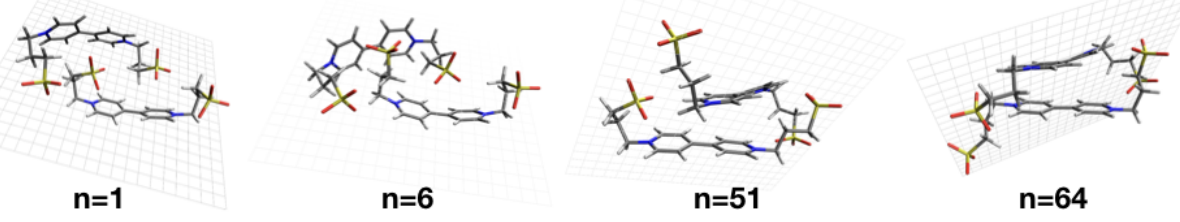

d) $\left[\mathrm{DQ} \cdot{ }^{\cdot+}\right]_{2}$
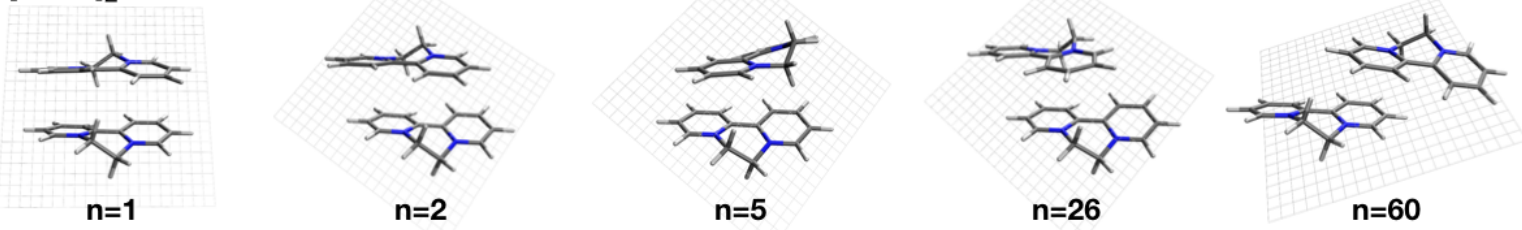

e) $\left[4,4^{\prime}-\mathrm{DMDQ} \cdot+\right]_{2}$
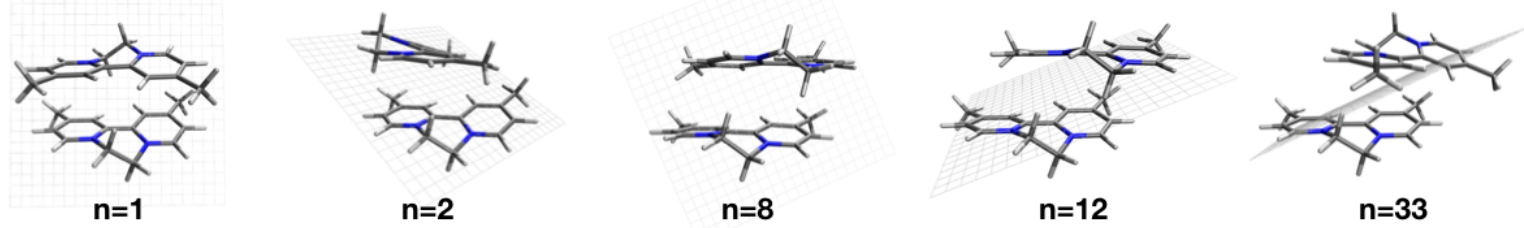

f) $\left[5,5^{\prime}-\mathrm{DMDQ} \cdot+\right]_{2}$

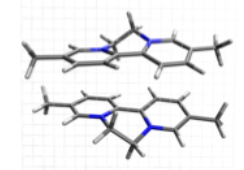

$n=1$
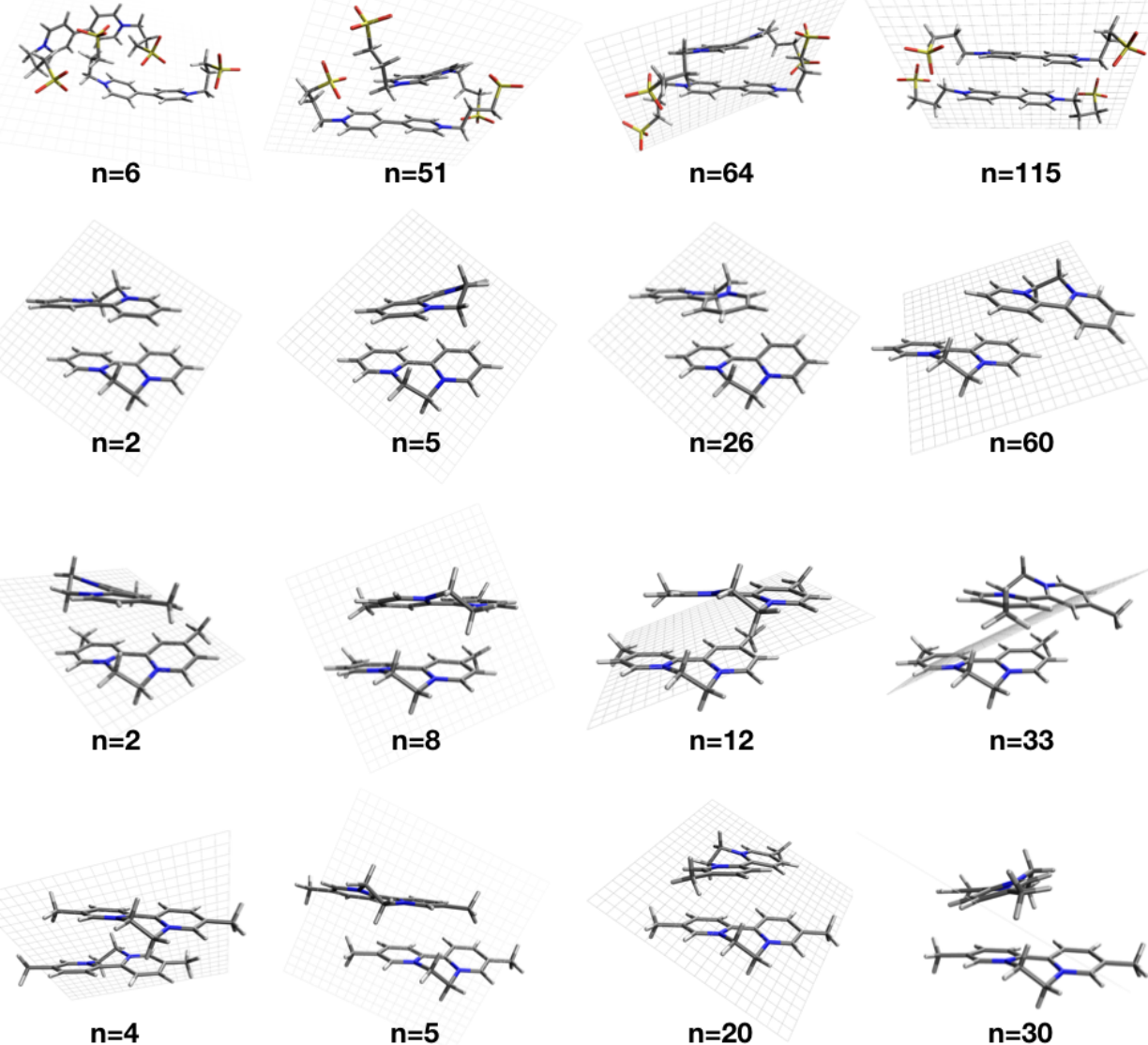

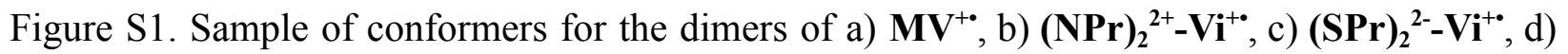

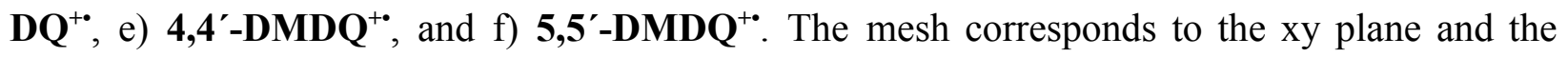
conformer number is indicated by the $n$ value. All structures are included in the set for further single-point DFT calculations. 
Table S1. Interaction energies in $\mathrm{kcal} \mathrm{mol}^{-1}$ calculated from single-point energies $\left(\Delta \mathrm{E}_{\mathrm{SP}}\right)$ with the $\omega$ B97X-D functional, def2-TZVP basis set, and SMD solvation model in water. Values in bold were selected for further DFT geometry optimization. For $(\mathrm{SPr})_{2}{ }^{2-}-\mathrm{Vi}^{+\bullet}$, the $\pi$-anion interactions are indicated with an asterisk $(*)$.

\begin{tabular}{|c|c|c|c|c|c|c|}
\hline ID & $\mathbf{M V}^{+\bullet}$ & $(\mathrm{NPr})_{2}{ }^{2+}-\mathrm{Vi}^{\mathbf{t}^{\bullet}}$ & $(\mathrm{SPr})_{2}{ }^{2-}-\mathrm{Vi}^{+\bullet}$ & $\mathbf{D Q}^{+\bullet}$ & $4,4^{\prime}-\mathrm{DMDQ}^{+\bullet}$ & 5,5'-DMDQ ${ }^{+\bullet}$ \\
\hline 1 & -13.3481 & -10.6055 & $-3.7296^{*}$ & -13.0231 & -17.3176 & -15.6198 \\
\hline 2 & -14.2258 & -11.0565 & $2.6704 *$ & -11.4900 & -14.3306 & -13.9453 \\
\hline 3 & -13.0738 & -11.1580 & $0.8960^{*}$ & -12.0033 & -14.2393 & -15.1382 \\
\hline 4 & -13.9056 & -10.4912 & $3.9465^{*}$ & -11.8116 & -14.3702 & -14.2734 \\
\hline 5 & -13.8567 & -11.2037 & $2.1851^{*}$ & -12.4403 & -14.1905 & -14.3352 \\
\hline 6 & -10.6289 & -11.3756 & $-4.6006^{*}$ & -11.7331 & -16.3145 & -14.9259 \\
\hline 7 & -11.3112 & -10.5935 & $2.7173^{*}$ & -12.0688 & -16.1832 & -13.1157 \\
\hline 8 & -10.7712 & -10.1507 & $-2.2600 *$ & -11.6029 & -14.6023 & -15.1815 \\
\hline 9 & -10.1195 & -11.5355 & $12.8531 *$ & -12.2802 & -14.4539 & -14.6805 \\
\hline 10 & -10.1834 & -11.3854 & $-3.2282 *$ & -10.0260 & -14.1576 & -14.5679 \\
\hline 11 & - & -10.8358 & -14.6717 & -10.7372 & -13.5424 & -13.9175 \\
\hline 12 & - & -6.6799 & -17.7220 & -10.3298 & -14.7995 & -12.8478 \\
\hline 13 & - & -12.2627 & -1.4349 & -9.6818 & -13.4455 & -11.9520 \\
\hline 14 & - & -9.5927 & -13.6519 & -7.7305 & -13.1224 & -12.2555 \\
\hline 15 & - & -12.2391 & $-4.6323 *$ & -6.7172 & -11.9770 & -11.3784 \\
\hline 16 & - & -12.2261 & 5.2082 & -5.1002 & -12.6490 & -9.6593 \\
\hline 17 & - & -12.0422 & -12.1995 & -4.4526 & -11.2559 & -9.1467 \\
\hline 18 & - & -11.8790 & -9.8101 & -4.5161 & -13.0642 & -9.5447 \\
\hline 19 & - & -11.4524 & -4.9479 & -4.1532 & -10.1017 & -8.6746 \\
\hline 20 & - & -9.8123 & -15.2905 & -1.7020 & -6.3241 & -5.5111 \\
\hline 21 & - & -11.1820 & - & - & - & - \\
\hline
\end{tabular}


Table S2. Interaction energies optimized $\left(\Delta \mathrm{E}_{\mathrm{OPT}}\right)$, and calibrated using the $\mathbf{D} \mathbf{Q}^{+\bullet}$ pimer $\left(\Delta \mathrm{E}_{\mathrm{CAL}}\right)$. Optimizations were performed with the $\omega$ B97X-D functional, def2-TZVP basis set, and SMD solvation model in water. Top projections for the respective DFT optimized pimers of $\mathbf{D Q}^{+\bullet}$,

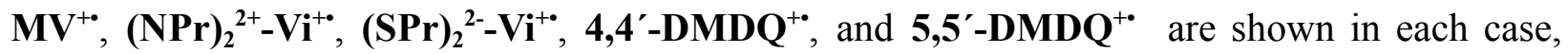
represented with light and dark monomers. ID numbers correspond to numeration shown in figure 8 in the paper and numbers in parenthesis to the table S1.

\begin{tabular}{|c|c|c|c|c|}
\hline ID & Dimer & $\begin{array}{c}\Delta \mathbf{E}_{\mathrm{OPT}} \\
\left(\mathrm{kcal}_{\mathrm{mol}}^{-1}\right)\end{array}$ & $\underset{\left(\mathrm{kcal}_{\mathrm{CAL}} \mathrm{mol}^{-1}\right)}{\Delta \mathrm{E}_{\mathrm{C}}}$ & $\begin{array}{c}\text { Optimized } \\
\text { structure }\end{array}$ \\
\hline $\begin{array}{c}1 \\
(1)\end{array}$ & {$\left[\mathbf{M V}^{+\bullet}\right]_{2}$} & -15.702 & -1.873 & \\
\hline $\begin{array}{c}\mathbf{2} \\
(2)\end{array}$ & {$\left[\mathbf{M V}^{+*}\right]_{2}$} & -15.914 & -2.085 & \\
\hline $\begin{array}{c}1 \\
(1)\end{array}$ & {$\left[(\mathbf{N P r})_{2}{ }^{2+}-\mathbf{V i}^{+\bullet}\right]_{2}$} & -15.829 & -2.000 & \\
\hline$\underset{(21)}{\mathbf{2}}$ & {$\left[(\mathbf{N P r})_{2}{ }^{2+}-\mathbf{V i}^{\mathbf{+}^{+\bullet}}\right]_{2}$} & -12.769 & 1.060 & \\
\hline $\begin{array}{c}1 \\
(18)\end{array}$ & {$\left[(\mathbf{S P r})_{2}{ }^{2-}-\mathbf{V i}^{\mathbf{+}^{+}}\right]_{2}$} & -16.817 & -2.988 & \\
\hline
\end{tabular}




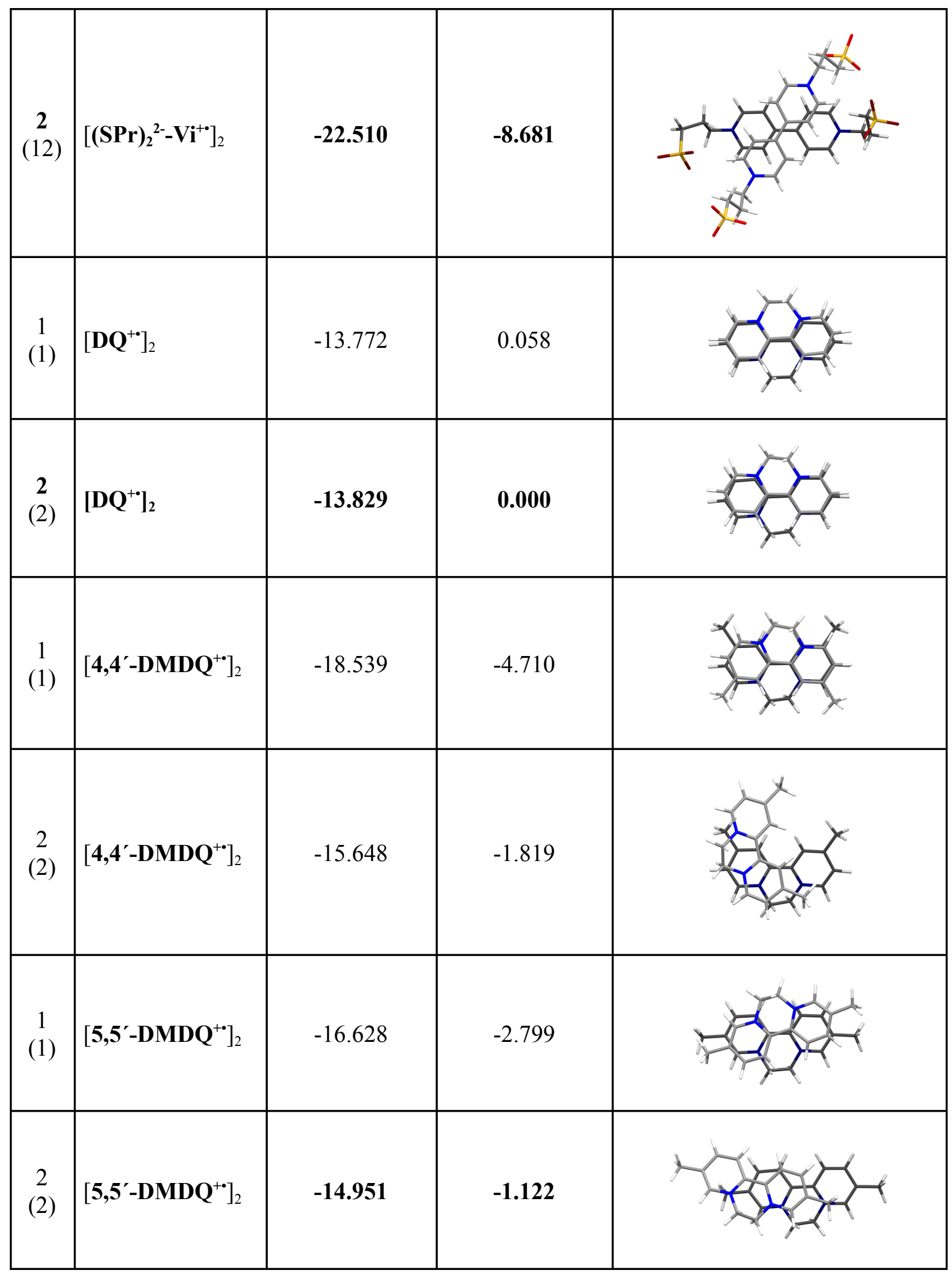

